\title{
High-gain amplification for femtosecond optical vortex with mode-control regenerative cavity
}

\author{
Shuiqin Zheng, ${ }^{1,2,3}$ Zhenkuan Chen, ${ }^{1,3}$ QInggang Lin, ${ }^{1}$ Yi CAI, ${ }^{1}$ XiaOWei Lu, ${ }^{1}$ \\ YANXIA GAO, ${ }^{1,4}$ ShIXIANG XU, ${ }^{1,}{ }^{*}$ AND DIANYUAN FAN ${ }^{3}$ \\ ${ }^{1}$ Shenzhen Key Lab of Micro-Nano Photonic Information Technology, College of Physics and Optoelectronics Engineering, Shenzhen University \\ Shenzhen, Guangdong 518060, China \\ ${ }^{2}$ Photonics Laboratory, Division of Computer, Electrical, and Mathematical Sciences and Engineering, King Abdullah University of Science and \\ Technology (KAUST), Thuwal 23955-6900, Kingdom of Saudi Arabia \\ ${ }^{3}$ SZU-NUS Collaborative Innovation Center for Optoelectronic Science \& Technology, Key Laboratory of Optoelectronic Devices and Systems of \\ Ministry of Education and Guangdong Province, Shenzhen University, Guangdong 518060, People's Republic of China \\ ${ }^{4}$ gyx@szu.edu.cn \\ *Corresponding author: shxxu@szu.edu.cn
}

Received XX Month XXXX; revised XX Month, XXXX; accepted XX Month XXXX; posted XX Month XXXX (Doc. ID XXXXX); published XX Month XXXX

\begin{abstract}
Ultra-intense femtosecond vortex pulses can provide an opportunity to investigate the new phenomena with orbital angular momentum (OAM) involved in extreme cases. This paper reports a high gain optical vortex amplifier for intense femtosecond vortex pulses generation. Traditional regeneration amplifiers can offer high gain for Gaussian mode pulses but cannot amplify optical vortex pulses while maintaining the phase singularity because of mode competition. Here, we present a regeneration amplifier with a ringshaped pump. By controlling the radius of the pump, the system can realize the motivation of $\mathrm{LG}_{0,1(-1)}$ mode and the suppression of Gaussian mode. Without seeds, the amplifier has a donut-shaped output containing two opposite OAM states simultaneously, as our prediction by simulation. If seeded a pulse of a topologic charge of 1 or -1 , the system will output an amplified LG,1(-1) mode pulse with the same topologic charge as the seed. To our knowledge, this amplifier can offer the highest gain as $1.45 \times 10^{6}$ for optical vortex amplification. Finally, we obtain a $1.8 \mathrm{~mJ}, 51$ fs compressed optical vortex seeded from a $2 \mathrm{~nJ}$ optical vortex. @ 2020 Optical Society of America
\end{abstract}

http://dx.doi.org/10.1364/PRJ.99.099999

\section{Introduction}

An optical vortex beam is a structured beam with a spiral phase $e^{-i l \phi}$ in the azimuthal direction $\phi$ perpendicular to the propagation direction [1-3]. These beams carrying an orbital angular momentum (OAM) of $l \hbar$ per photon have opened a route to many promising applications, such as optical communication [4-8], laser ablation [9-12] and micromanipulation [13-15]. These applications can benefit from technologies of optical vortex generation which can be divided into external modulation outside the cavity [16-20] and direct generation within the cavity [21-23]. Direct generation may face multi-mode superposition which can be divided into coherent superposition and incoherent superposition [24,25]. With the increase of the peak power of the optical vortex, novel phenomena are emerging. The ultra-intense ultra-short optical vortex pulse can provide an opportunity to investigate the new phenomena with OAM involved in extreme cases [26-29]. The spiral phase can be introduced to the ultra-short Gaussian mode laser by external modulation through spiral phase plate $[19,28]$, fork grating [30-34], and spatial light modulator [11, 35]. Unfortunately, such methods for ultra-intense cases pose a significant challenge owing to technical difficulties like optical element damage and the dispersion hindering the synthesis of a high-power broadband optical vortex. As the other way for the generation of ultrashort vortices, the direct generation from the mode-locked laser was proved [36, 37].
However, this way also cannot generate ultra-short vortices with ultrahigh intensity.

Looking back to the development history of ultra-short laser, we can see that amplification technology plays a very important role in the generation of ultra-short pulses with ultra-high intensity [38, 39]. Regenerative amplification can provide a large gain [40] and chirped pulse amplification (CPA) can help to reduce the damage and nonlinear effects [41]. Ti: Sapphire (Ti: S) based amplifier plays an important role in delivering high peak power [42]. For the ultra-intense ultra-short optical vortex generation, the blessing of amplification technologies may be indispensable. Compared with conventional ultra-short pulse amplifiers, the input and output of an optical vortex amplifier (OVA) should be both optical vortices with the same topologic charge, and some efforts have been made. Single-pass of two Nd: YAG rod can amplify optic vortex to several times [43]. A multi-pass amplifier for femtosecond optical vortex pulses in a CPA system with a gain level of 10 was demonstrated [30]. Optical parametric amplification for ultrashort optical-vortex pulses generation makes a gain level up to $10^{2}$ [44-46]. As far as we know, currently, there are no OVA can reach a gain level of $10^{6}$. Because these amplifiers cannot make the vortex beam pass through the gain medium enough times.

In this paper, we develop a high gain OVA whose gain reaches $10^{6}$ level based on regenerative amplification. Firstly, we show that the conventional regenerative amplifier (RA) could not amplify the optical 
vortex and the vortex seed will degenerate to a Gaussian beam because of the mode competition. Then, to overcome this problem, we pump the RA with the ring-shaped laser for controlling mode competition. Our simulations show choosing a right pump radius can suppress Gaussian mode generation and increase the gain of target Laguerre-Gaussian (LG) mode. Therefore, one LG mode can be amplified from a weak vortex seed while maintaining the topological charge (TC) and the spiral phase. Finally, an OVA for $\mathrm{LG}_{0,1(-1)}$ mode has been built experimentally with an amplification gain up to $1.45 \times 10^{6}$ for a $2 \mathrm{~nJ}$ vortex seed. Base on the OVA, the $51 \mathrm{fs}, 1.8 \mathrm{~mJ}$ compressed optical vortex has been obtained.

\section{Mode competition in a regenerative amplifier}

Regenerative amplification has the highest gain among all amplification technologies because the pulse can pass through the gain medium enough times. It is well known that an RA is an active laser cavity which works as a $Q$-switched laser operating at cavity dumping mode with a weak seed instead of oscillation from noise. $\mathrm{LG}_{p, l}$ modes are the selfconsistent modes of a cylindrically symmetrical cavity, where $p$ is the index of the radial order and $l$ is the TC. And the fundamental mode is the $\mathrm{LG}_{0,0}$, which is also named Gaussian mode. The seed of RA should be matched to the most competitive self-consistent mode (usually a Gaussian mode) of the cavity for the effective amplification. Traditionally, a Gaussian mode seed is injected into the cavity, and we can obtain an amplified Gaussian output. In spite that the mode mismatching occurs, the seed can be still amplified, but be pulled towards the Gaussian mode. As shown in Fig. 1, a 2 nJ vortex pulse with $l=1$ evolves into Gaussian mode during the amplification. Unfortunately, the Gaussian mode does not carry any orbital angular momentum, which means we cannot amplify vortex pulses directly with a conventional RA. Obviously, the reason for this is the mode competition.

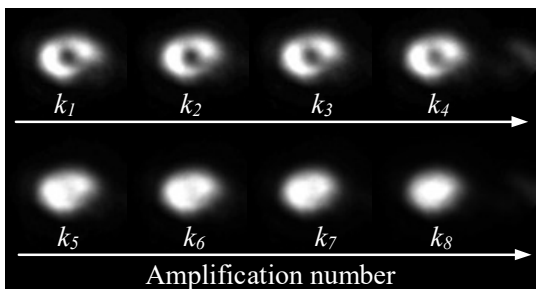

Fig. 1 Evolution of an optical vortex seed with $l=1$ in a conventional RA, and the amplification number $k_{n}=4 n-3$.

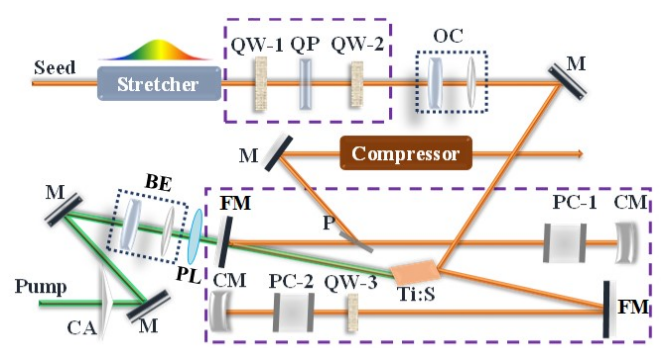

Fig. 2 The setup of the proposed RA. QW: quarter-wave plates; QP: Qplate; OC: optical coupling systems; M: plane mirrors; CM: concave mirror, $\mathrm{R}=-1 \mathrm{~m}$; PM: fold mirror, $\mathrm{R}=0.9 \mathrm{~m}$; PC: Pock cell; BE: beam expander; P: polarizer; PL: pump lens, $\mathrm{f}=30 \mathrm{~cm}$; $\mathrm{CA}$ : convex axicon, base angle of $0.5^{\circ}$; Ti:S: Ti:Sapphire, length of $25.4 \mathrm{~mm}$.

In order to overcome the mode competition and realize the amplification of vortex pulses, we need the net gain of the target LG mode carrying target OAM greater than that of other modes, particularly to the Gaussian mode. This is also the key to the vortex emission within the cavity, and some methods have been reported, such as spot-defect mirror [47] and a ring-shaped pump [36, 48, 49]. Here, we choose the ring-shaped pump for the suppression of Gaussian mode and excitation of the vortex modes. And our setup is reformed from a commercial $1 \mathrm{kHz}-800 \mathrm{~nm}$ Ti:SRA (Coherence Inc., Legend-Elite-He). As shown in Fig.2, the ring-shaped pump is realized by using a convex axicon (CA, Thorlabs, AX2505-A) to modulate a $50 \mathrm{~ns}, 15 \mathrm{~mJ}, 532 \mathrm{~nm}$ beam [50]. Besides, a variable beam expander (BE) is required to adjust the pump radius (the pump radius is inversely proportional to the beam expansion ratio), and the expansion ratio is set at 3.5 for the motivating of the $\mathrm{LG}_{0,1(-1)}$ mode. This RA without BE and CA is optimized in Gaussian mode and all components do not have further adjustment after the optimization except the pump-related components.

For an active cavity without seed, its initial signal starts from the noise and the final output mode is determined by the gains of modes. The differences in these gains can be reflected in the mode probabilities of the output. For our RA, the concerned LG modes in the output mainly include $\mathrm{LG}_{0,0}, \mathrm{LG}_{0,1}$ and $\mathrm{LG}_{0,-1}$. The purity $\boldsymbol{\rho}_{p, l}$ of $\mathrm{LG}_{p, l}$ in the optical field, $E$ for a coherent superposition can be express as

$$
\boldsymbol{\rho}_{p, l}=\frac{\left\langle\boldsymbol{E} \mid \boldsymbol{L} \boldsymbol{G}_{p, l}\right\rangle\left\langle\boldsymbol{L} \boldsymbol{G}_{p, l} \mid \boldsymbol{E}\right\rangle}{\langle\boldsymbol{E} \mid \boldsymbol{E}\rangle\left\langle\boldsymbol{L} \boldsymbol{G}_{p, l} \mid \boldsymbol{L} \boldsymbol{G}_{p, l}\right\rangle}
$$

where the inner product $\langle\boldsymbol{B} \mid \boldsymbol{A}\rangle$ of two fields $A$ and $B$ is defined as $\iint \boldsymbol{B}^{*}(\boldsymbol{x}, \boldsymbol{y}) \boldsymbol{A}(\boldsymbol{x}, \boldsymbol{y}) \boldsymbol{d} \boldsymbol{x} \boldsymbol{d} \boldsymbol{y}$. We simulate the laser oscillation from noise with the different radiuses of ring-shaped pump or different expansion ratios of BE using the method proposed in Ref. [51]. Firstly, we set the expansion ratio of $\mathrm{BE}$ as 4 . Our simulations start with different noises each time and we can obtain the different outputs oscillating from the different noises. For a output, we can get the corresponding purities of $\mathrm{LG}_{0,1}, \mathrm{LG}_{0,-1}$ and LG0, 0 as $\left(\boldsymbol{\rho}_{\mathbf{0 , 1}}, \boldsymbol{\rho}_{\mathbf{0},-\mathbf{1}}, \boldsymbol{\rho}_{\mathbf{0}, \mathbf{0}}\right)$. We simulate 1000 oscillations from different noises and the purities of the 1000 outputs are obtained. As shown in Fig. 3 (a), the $\left(\boldsymbol{\rho}_{\mathbf{0 , 1}}, \boldsymbol{\rho}_{\mathbf{0},-\mathbf{1}}, \boldsymbol{\rho}_{\mathbf{0}, \mathbf{0}}\right)$ are plotted in a three-dimensional coordinate, while the black star is for the average mode purities or mode probabilities $\left(\overline{\boldsymbol{\rho}}_{\mathbf{0 , 1}}, \overline{\boldsymbol{\rho}}_{\mathbf{0},-\mathbf{1}}, \overline{\boldsymbol{\rho}}_{\mathbf{0}, \mathbf{0}}\right)$. And can be seen from Fig.3(a), the $\mathrm{LG}_{0,1}$, and $\mathrm{LG}_{0,-1}$ start to appear, but $\mathrm{LG}_{0,0}$ is not well suppressed. When the expansion ratio is set as 3 , the $L_{0}, 0$ is well suppressed $\left(\boldsymbol{\rho}_{\mathbf{0}, \mathbf{0}} \rightarrow \mathbf{0}\right)$, as shown in Fig.3(c). However, the $\mathrm{LG}_{0,1}$, and $\mathrm{LG}_{0,-1}$ are not very well motivated because the pump radius is too large. However, if the expansion ratio is 3.5 , the $\mathrm{LG}_{0,1}$, and $\mathrm{LG}_{0,-1}$ is well motivated while $\mathrm{LG}_{0,0}$ is well suppressed, as shown in Fig.3 (b). The purities lay near the blue line which represents $\boldsymbol{\rho}_{\mathbf{0 , 1}}+\boldsymbol{\rho}_{\mathbf{0 , - 1}}=\mathbf{1}$, and mode probabilities is near the center of the blue line. Because the ringshaped pump is not selective between $\mathrm{LG}_{0,1}$, and $\mathrm{LG}_{0,-1}$, so the gains for these modes are the same, and we cannot select a unique vortex mode from the series of self-consistent LG modes by the ring-shaped pumped cavity. Consequently, the system generates the two modes with the same possibility in the absence of external seeds.
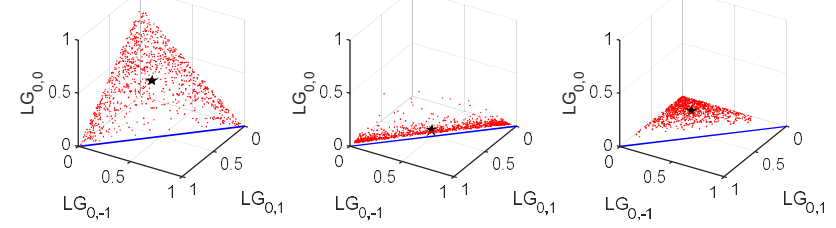

Fig. 3 Simulations of laser oscillations from noises with the different ring-shaped pump radiuses. Expansion ratios are 4(a), 3.5(b) and 3(c).

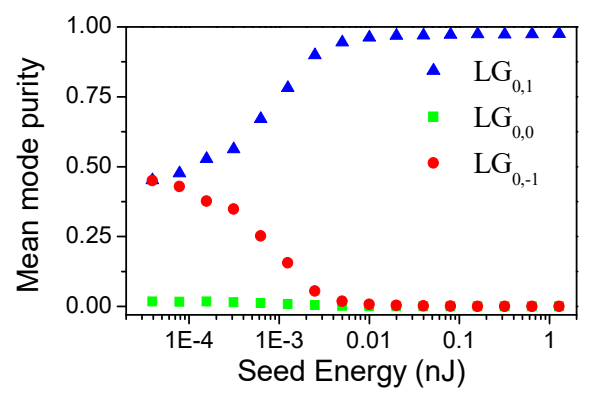

Fig. 4 Simulation of vortex amplification with different seed energies

For the RA, the final output depends on not only the gain of each mode but also the mode and energy of the input seed. We simulate the RA seeding with vortex pulse with $l=1$. For different seed energies, our simulation also runs 1000 times and the mode probabilities are 
obtained. As shown in Fig.4, if the seed is weak enough, $\mathrm{LG}_{0,1}$ and $\mathrm{LG}_{0,-1}$ are the two most possible modes to survive with the same possibility of $45 \%$. At the same time, the $\mathrm{LG}_{0,0}$ mode is well suppressed. The $\mathrm{LG}_{0,1}$ mode purity increases with the increase of seed energy while $\mathrm{LG}_{0,-1}$ mode purity decreases. If the seed energy rises to $0.01 \mathrm{~nJ}$, the purity goes up to $96 \%$ for $\mathrm{LG}_{0,1}$, but drops to $0.8 \%$ for $\mathrm{LG}_{0,-1}$, while $\mathrm{LG}_{0,0}$ always keeps its purity less than $0.12 \%$. Our simulations also find that, if the topological charge of the seed changes to -1 instead of 1 , the high probability mode will change to $\mathrm{LG}_{0,-1}$, instead of $\mathrm{LG}_{0,1}$.

\section{Experimental results and discussions}

In our experiments, we firstly align $\mathrm{BE}$ for matching the radius of ringshaped pump for the motivation of $\mathrm{LG}_{0,1(-1)}$ and the suppression of $\mathrm{LG}_{0,0}$. The expansion ratio of $\mathrm{BE}$ is chosen at 3.5 according to our simulation. Fig. 5(a) shows the ring-shaped pump at one of the Ti:S surfaces. Correspondingly, the output beam profile from the RA without seeding is shown in Fig. 5(b). The donut-shaped distribution of the output implies that $\mathrm{LG}_{0,0}$ has been successfully suppressed. The Dammannvortex grating can be used to check the TC of the output by measuring whether the pattern on the corresponding diffraction order is solid [52]. Fig. 5(c) is the phase structure of our Dammann-vortex grating, which is loaded into a spatial light modulator. Fig.5 (d) shows the simulated farfield diffraction patterns with parallel illumination. By determining whether the spots on the green box are solid, we can find out whether the beam contains an OAM component with $l=0$, while the blue box and the red box for $l=+1$ and -1 . Figure 5(e) presents the measured far-field spots after Dammann-vortex grating from the output of the unseeded RA. A very bright solid spot in the center is an exception, which is from the strong zero-order diffraction. Other solid spots only locate within the blue and red boxes, which means the output beam includes both $\mathrm{LG}_{0}$, 1 and $L_{0,-1}$ modes.

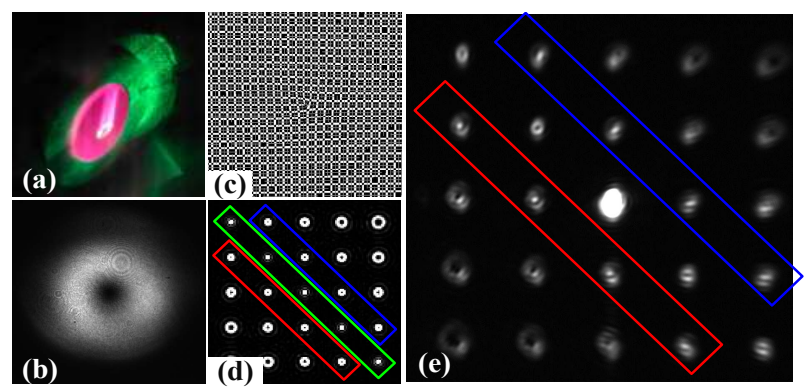

Fig. 5(a) The ring-shaped pump on one of the Ti:S surfaces, (b) the donut-shaped output from the unseeded RA, (c) the phase structure of our Dammann-vortex grating and (d) the corresponding far-field with parallel illumination, and (e) the measured far-field illuminated by the output of the unseeded RA

Our vortex seed with $l=1$ is converted from a Gaussian mode pulse from a mode-locked oscillator with a $q$-plate (QP) and two achromatic quarter-wave plates $(\mathrm{QW}-1$ and $\mathrm{QW}-2)$, as shown in Fig.2. And the final seed before the incident to the system has the pulse energy of $2 \mathrm{~nJ}$. And we check the TC of the seed by a cylindrical lens [53]. Fig. 6(a) is the recorded spatial profile of the seed focused by a cylindrical lens, which features one dark stripe across the beam spot, showing the seed has a TC of 1 . The stripe orientation depends on whether the TC is positive or negative. Correspondingly, the amplified output is recorded as Fig. 6(b), which also presents a donut-shaped profile. The corresponding far-field distribution after Dammann-vortex grating is shown in Fig.6(c). Compared with Fig. 5(e), its solid spots only exist inside the blue box. This means the output contains $\mathrm{LG}_{0,1}$ mode only and have the same TC with the seed. Then we rotate simultaneously $\mathrm{QW}-1$ and $\mathrm{QW}-2$ with an angle of $90^{\circ}$ to change the TC value of the seed from 1 to -1. Compare with Fig. 6(a), the cylindrical lens focused pattern of the seed changed the orientation of the tilted dark stripe, as shown in Fig. 7(a). This implies the seed changes to an opposite TC. As shown in Fig. 7(b), the amplified output seeded with $l=-1$, has a similar profile as that in Fig. 5(b) and Fig.6(b). Compare with Fig. 6(c), Fig. 7(c) confirms further the output has changed its TC value from +1 to -1 , showing our RA can keep the output maintaining the same TC from the seed. From Fig. 5(e), the rings around the spots are oval and disconnected on the minor axis sides. We infer that they result from the astigmatisms due to the slight misalignment of the Fourier-lens after the Dammann-vortex grating. The slightly oval rings on both sides of the red and blue boxes in Fig.6(c) and Fig. 7(c) are also caused by the astigmatism.

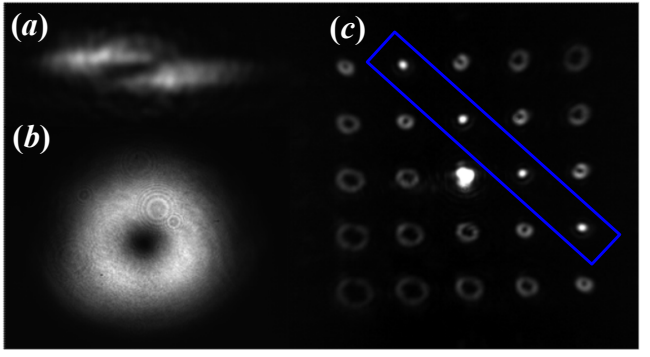

Fig. 6 The recorded spatial intensities of the seed with $l=1$ focused by a cylindrical lens (a), the output spatial intensity distribution (b) and the far-field distribution after Dammann-vortex grating

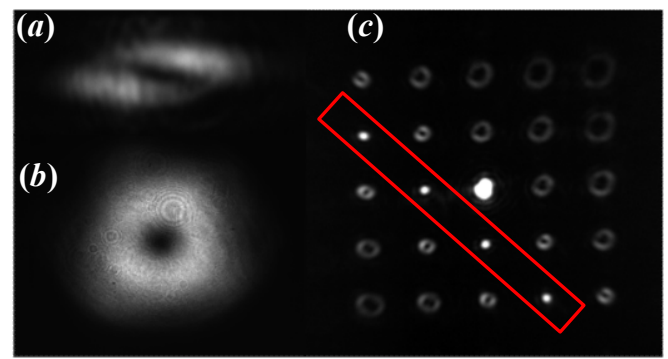

Fig. 7. The recorded spatial intensities of the seed with $l=-1$ focused by a cylindrical lens (a), the output spatial intensity distribution (b) and the far-field distribution after Dammann-vortex grating
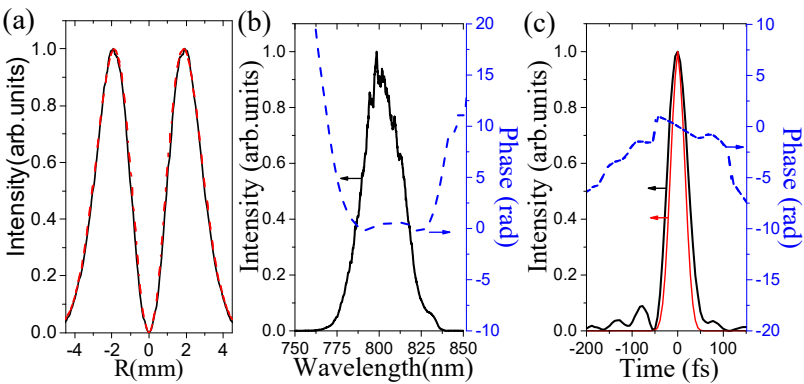

Fig. 8 (a) Spatial cross-section intensity of the amplified $\mathrm{LG}_{0,1}$ vortex: the average from the different orientations (black line) and theoretical fitting (dashed red line); (b) the spectral intensity and phase of the amplified LG0, 1 pulse; (c)The temporal intensity and phase of the amplified $\mathrm{LG}_{0,1}$ pulse (black and blue line) and the temporal intensity of corresponding Fourier-transform-limited pulse (red line).

From Fig.6 (b), we can get different cross-section intensity distributions across the center along with different directions, then we obtain the average cross-section from the orientations, as shown as the black line in Fig. 8(a). The dashed red line is the theoretical fit by $\mathrm{LG}_{0,1}$, which agrees with the average data very well. For the amplified $\mathrm{LG}_{01}$ pulse, the spiral phase distribution is verified by the Dammann-vortex grating and we can determine the phase term as $\mathrm{e}^{-i l \phi}$ with $l=1$. The amplitude term can be obtained from the captured intensity. With these two terms, the mode purity of $\mathrm{LG}_{01}$ from the output is $95 \%$ according to Eq. (1). This means our RA output high-quality $\mathrm{LG}_{0,1}$ pulse. Compared with the same RA cavity with the Gaussian pump, our RA with a ring-shaped pump has smaller 
gain under the same pump power because of the deduction of pump density. Smaller net gain will result in larger amplification pass number $N$ for saturated output and smaller output power. According to our experiment, the $N$ for saturated amplification is 53 for the ring-shaped pump and it is 27 for the Gaussian pump under the same pump energy of $15 \mathrm{~mJ}$. Before entering the compressor, the output pulse energies are $2.9 \mathrm{~mJ}$ for the ring-shaped pump and $4.5 \mathrm{~mJ}$ for the Gaussian pump. From this, the gain of our OVA is $1.45 \times 10^{6}$. And the corresponding output energies are $1.8 \mathrm{~mJ}$, and $3.2 \mathrm{~mJ}$ after compression. The pulse durations of the outputs have been measured with SPIDER. The spectral intensity and phase of the amplified $\mathrm{LG}_{0,1}$ pulse are shown in Fig.8(b). Figure 8(c) shows the $\mathrm{LG}_{0,1}$ pulse duration is about $51 \mathrm{fs}$ with the black line, which is wider than 45.3 fs of the Gaussian pulse. This is because the larger $N$ implies to induce larger high order material dispersion which cannot be compensated by a compressor. However, the red line in Fig. 8(c) reveals the spectrum of the amplified $\mathrm{LG}_{0,1}$ pulse can support a Fourier-transform-limited pulse with a pulse duration as short as 35 fs.

\section{Conclusions}

Summarily, in order to overcome the mode competition problem for the amplification of optical vortex in RA, we develop a high gain OVA with the mode-control cavity. The RA is pumped with the ring-shaped laser for controlling mode competition. Our simulation shows, by choosing a right radius of the pump can suppress the generation of Gaussian mode and increase the gain of $\mathrm{LG}_{0}, 1(-1)$ mode. In our experiment, an RA for $\mathrm{LG}_{0,1(-1)}$ mode is built. And it verifies that, without a seeding, the amplifier has a donut-shaped output containing two opposite OAM states simultaneously. By seeding pulses with the topologic charge of 1 , the system will output amplified $\mathrm{LG}_{0,1}$ mode pulses with the same topologic charge to the seed. The sign of the output topological charge can be changed accordingly by changing that of the vortex seed. This shows our OVA whose gain is $1.45 \times 10^{6}$ for $2 \mathrm{~nJ}$ seed can maintain the topological charge and the spiral phase of the vortex seed during amplification. Finally, a $1.8 \mathrm{~mJ}, 51 \mathrm{fs}$ optical vortex after compression is obtained.

Funding. The National Natural Science Foundation of China (NSFC) (61775142, 61490710,61705132); Shenzhen basic research projecton subject layout (JCYJ20170412105812811); and Shenzhen basic research projects (JCYJ20190808164007485, JCYJ20190808 115601653)

Disclosures. The authors declare no conflicts of interest.

\section{REFERENCES}

1.A. M. Yao, and M. J. Padgett, "Orbital angular momentum: origins, behavior and applications," Adv. Opt. Photonics 3, 161204 (2011)

2. L. Allen, M. W. Beijersbergen, R. J. C. Spreeuw, and J. P. Woerdman, "Orbital angular momentum of light and the transformation of Laguerre-Gaussian laser modes," Phys. Rev. A 45, 8185-8189 (1992).

3. S. Franke-Arnold, L. Allen, and M. Padgett, "Advances in optical angular momentum," Laser Photonics Rev. 2, 299-313 (2008).

4. A. E. Willner, H. Huang, Y. Yan, Y. Ren, N. Ahmed, G. Xie, C. Bao, L. Li, Y. Cao, Z. Zhao, J. Wang, M. P. J. Lavery, M. Tur, S. Ramachandran, A. F. Molisch, N. Ashrafi, and S. Ashrafi, "Optical communications using orbital angular momentum beams," Adv. Opt. Photonics 7, 66-106 (2015).

5. J. Wang, J. Yang, I. M. Fazal, N. Ahmed, Y. Yan, B. Shamee, A. E.Willner, K. Birnbaum, J. Choi, B. Erkmen, S. Dolinar, and M.Tur, "25.6-bit/s/Hz spectral efficiency using 16-QAM signals over pol-muxed multiple orbital-angular-momentum modes," IEEE Photonics Conference 58, 587-588 (2011).

6. T. Lei, M. Zhang, Y. Li, P. Jia, G. N. Liu, X. Xu, Z. Li, C. Min, J. Lin, C. Yu, H. Niu, and X. Yuan, "Massive individual orbital angular momentum channels for multiplexing enabled by Dammann gratings," Light-Sci. Appl. 4, e257 (2015).

7. J. Wang, "Advances in communications using optical vortices," Photonics Res. 4, B14-B28 (2016).

8. E. Stegenburgs, A. Bertoncini, A. Trichili, M. S. Alias, T. K. Ng, M. Alouini, C. Liberale, and B. S. Ooi, "Near-Infrared OAM Communication Using 3D-Printed Microscale Spiral Phase Plates," IEEE Commun. Mag. 57, 65-69 (2019).

9. J. Hamazaki, R. Morita, K. Chujo, Y. Kobayashi, S. Tanda, and T. Omatsu, "Optical-vortex laser ablation," Opt. Express 18, 2144 (2010).

10. T. Omatsu, K. Chujo, K. Miyamoto, M. Okida, K. Nakamura, N. Aoki, and R. Morita, "Metal microneedle fabrication using twisted light with spin," Opt. Express 18, 17967-17973 (2010). 11. B. Wetzel, C. Xie, P. Lacourt, J. M. Dudley, and F. Courvoisier, "Femtosecond laser fabrication of micro and nano-disks in single layer graphene using vortex Bessel beams," Appl. Phys. Lett. 103, 241111 (2013)

12. F. Courvoisier, R. Stoian, and A. Couairon, "[INVITED] Ultrafast laser micro- and nano-processing with nondiffracting and curved beams," Opt. Laser Technol. 80, 125-137 (2016).

13. S. R. Lee, J. Kim, S. Lee, Y. Jung, J. K. Kim, and K. Oh, "All-silica fiber Bessel-like beam generator and its applications in longitudinal optical trapping and transport of multiple dielectric particles," Opt. Express 18, 25299-25305 (2010).

14. C. Liu, Z. Guo, Y. Li, X. Wang, and S. Qu, "Manipulating ellipsoidal micro-particles by femtosecond vortex tweezers," J. Optics-UK 17, 35402 (2015).

15. N. Eckerskorn, R. Bowman, R. A. Kirian, S. Awel, M. Wiedorn, J. Küpper, M. J. Padgett, H. N. Chapman, and A. V. Rode, "Optically Induced Forces Imposed in an Optical Funnel on a Stream of Particles in Air or Vacuum," Phys. Rev. Appl. 4, 064001 (2015). 16. P. Chen, B. Wei, W. Ji, S. Ge, W. Hu, F. Xu, V. Chigrinov, and Y. $\mathrm{Lu}$, "Arbitrary and reconfigurable optical vortex generation: a high-efficiency technique using director-varying liquid crystal fork gratings," Photonics Res. 3, 133-139 (2015).

17. J. Xin, K. Dai, L. Zhong, Q. Na, and C. Gao, "Generation of optical vortices by using spiral phase plates made of polarization dependent devices," Opt. Lett. 39, 1984 (2014).

18. Y. Tokizane, K. Oka, and R. Morita, "Supercontinuum optical vortex pulse generation without spatial or topological-charge dispersion," Opt. Express 17, 14517 (2009).

19. N. Matsumoto, T. Ando, T. Inoue, Y. Ohtake, N. Fukuchi, and T. Hara, "Generation of high-quality higher-order LaguerreGaussian beams using liquid-crystal-on-silicon spatial light modulators," J. Opt. Soc. Am. A 25, 1642-1651 (2008).

20. K. J. Moh, X. C. Yuan, D. Y. Tang, W. C. Cheong, L. S. Zhang, D. K. Y. Low, X. Peng, H. B. Niu, and Z. Y. Lin, "Generation of femtosecond optical vortices using a single refractive optical element," Appl. Phys. Lett. 88, 91103 (2006).

21. M. Okida, T. Omatsu, M. Itoh, and T. Yatagai, "Direct generation of high power Laguerre-Gaussian output from a diode-pumped Nd:YVO(4) 1.3-mum bounce laser," Opt. Express 15, 7616-7622 (2007).

22. D. J. Kim, and J. W. Kim, "Direct generation of an optical vortex beam in a single-frequency Nd:YVO4 laser," Opt. Lett. 40, 399-402 (2015).

23. Y. Zhao, Z. Wang, H. Yu, S. Zhuang, H. Zhang, X. Xu, J. Xu, X. Xu, and J. Wang, "Direct generation of optical vortex pulses," Appl. Phys. Lett. 101, 31113 (2012).

24. I. A. Litvin, S. Ngcobo, D. Naidoo, K. Ait-Ameur, and A. Forbes, "Doughnut laser beam as an incoherent superposition of two petal beams," Opt. Lett. 39, 704 (2014).

25. D. Naidoo, K. Aït-Ameur, M. Brunel, and A. Forbes, "Intracavity generation of superpositions of Laguerre-Gaussian beams," Applied Physics B 106, 683-690 (2012).

26. D. Pengel, S. Kerbstadt, D. Johannmeyer, L. Englert, T. Bayer, 
and M. Wollenhaupt, "Electron Vortices in Femtosecond Multiphoton Ionization," Phys. Rev. Lett. 118, 53003 (2017). 27. A. Leblanc, A. Denoeud, L. Chopineau, G. Mennerat, P. Martin, and F. Quéré, "Plasma holograms for ultrahigh-intensity optics," Nat. Phys. 13, 440-443 (2017).

28. D. Gauthier, P. R. Ribič, G. Adhikary, A. Camper, C. Chappuis, R. Cucini, L. F. DiMauro, G. Dovillaire, F. Frassetto, R. Géneaux, P. Miotti, L. Poletto, B. Ressel, C. Spezzani, M. Stupar, T. Ruchon, and G. De Ninno, "Tunable orbital angular momentum in highharmonic generation," Nat. Commun. 8, 1-7 (2017).

29. L. Rego, K. M. Dorney, N. J. Brooks, Q. L. Nguyen, C. Liao, J. San Román, D. E. Couch, A. Liu, E. Pisanty, M. Lewenstein, L. Plaja, H. C. Kapteyn, M. M. Murnane, and C. Hernández-García, "Generation of extreme-ultraviolet beams with time-varying orbital angular momentum," Science 364, eaaw9486 (2019).

30. Y. Lin, Y. Nabekawa, and K. Midorikawa, "Generation of intense femtosecond optical vortex pulses with blazed-phase grating in chirped-pulse amplification system of Ti:sapphire laser," Appl. Phys. B. 122, 280 (2016).

31. K. Bezuhanov, A. Dreischuh, G. G. Paulus, M. G. Schatzel, and H. Walther, "Vortices in femtosecond laser fields," Opt. Lett 29, 1942-1944 (2004).

32. Z. Guo, S. Qu, and S. Liu, "Generating optical vortex with computer-generated hologram fabricated inside glass by femtosecond laser pulses," Opt. Commun. 273, 286-289 (2007). 33. A. Schwarz, and W. Rudolph, "Dispersion-compensating beam shaper for femtosecond optical vortex beams," Opt. Lett. 33, 2970-2972 (2008)

34. I. G. Mariyenko, J. Strohaber, and C. J. G. J. Uiterwaal, "Creation of optical vortices in femtosecond pulses," Opt. Express 13, 7599 (2005).

35. Ó. Martínez-Matos, J. A. Rodrigo, M. P. Hernández-Garay, J. G. Izquierdo, R. Weigand, M. L. Calvo, P. Cheben, P. Vaveliuk, and L. Bañares, "Generation of femtosecond paraxial beams with arbitrary spatial distribution," Opt. Lett. 35, 652 (2010).

36. Y. Zhang, H. Yu, H. Zhang, X. Xu, J. Xu, and J. Wang, "Self-modelocked Laguerre-Gaussian beam with staged topological charge by thermal-optical field coupling," Opt. Express 24, 5514 (2016). 37. N. Li, J. Huang, B. Xu, Y. Cai, J. Lu, L. Zhan, Z. Luo, H. Xu, Z. Cai, and W. Cai, "Direct generation of an ultrafast vortex beam in a CVD-graphene-based passively mode-locked Pr:LiYF4 visible laser," Photonics Res. 7, 1209-1213 (2019).

38. A. Rundquist, C. Durfee, Z. Chang, G. Taft, E. Zeek, S. Backus, M.M. Murnane, H.C. Kapteyn, I. Christov, and V. Stoev," Ultrafast laser and amplifier sources," Appl. Phys. B 65, 161 (1997).

39. W. S. Brocklesby, "Progress in high average power ultrafast lasers," Eur. Phys. J. Spec. Top. 224, 2529(2015)

40. S. Backus, C. G. Durfee III, M. M. Murnane, and H. C. Kapteyn, "High power ultrafast lasers," Rev. Sci. Instrum. 69, 1207-1223 (1998).

41. D. Strickl, and G. Mourou, "Compression of amplified chirped optical pulses,". Opt. Commun. 56, 219-221 (1985).

42. J. Rothhardt, S. Hädrich, J. C. Delagnes, E. Cormier, and J. Limpert, "High Average Power Near-Infrared Few-Cycle Lasers," Laser Photonics Rev. 11, 1700043 (2017).

43. X. Chen, C. Chang, Z. Lin, P. Ding, and J. Pu, "High-Energy Nanosecond Optical Vortex Output From Nd:YAG Amplifiers," IEEE Photonic Tech. L. 28, 1271-1274 (2016).

44. K. Yamane, Y. Toda, and R. Morita, "Ultrashort optical-vortex pulse generation in few-cycle regime," Opt. Express 20, 1898618993 (2012).

45. A. Berzanskis, A. Matijosius, A. Piskarskas, V. Smilgevicius, and A. Stabinis, "Conversion of topological charge of optical vortices in a parametric frequency converter," Opt. Commun. 140, 273-276 (1997).

46. J. Qian, Y. Peng, Y. Li, P. Wang, B. Shao, Z. Liu, Y. Leng, and R. $\mathrm{Li}$, "Femtosecond mid-IR optical vortex laser based on optical parametric chirped pulse amplification," Photonics Res. 8, 421$425(2020)$.

47. S. Tan, C. Zhou, A. Shirakakwa, K. Ueda, and J. Li, "Vortex Ti:Sapphire laser by using an intracavity spot-defect spatial filter," Opt. Laser Technol. 96, 76-80 (2017).

48. D. J. Kim, and J. W. Kim, "Direct generation of an optical vortex beam in a single-frequency Nd:YVO4 laser," Opt. Lett. 40, 399-402 (2015).

49. J. W. Kim, and W. A. Clarkson, "Selective generation of Laguerre-Gaussian (LGon) mode output in a diode-laser pumped Nd:YAG laser," Opt. Commun. 296, 109-112 (2013).

50. M. Wei, W. Shiao, and Y. Lin, "Adjustable generation of bottle and hollow beams using an axicon," Opt. Commun. 248, 7-14 (2005).

51. A. Hu, J. Lei, P. Chen, Y. Wang, and S. Li, "Numerical investigation on the generation of high-order Laguerre-Gaussian beams in end-pumped solid-state lasers by introducing loss control," Appl. Opt. 53, 7845-7853 (2014).

52. N. Zhang, X. C. Yuan, and R. E. Burge, "Extending the detection range of optical vortices by Dammann vortex gratings," Opt. Lett. 35, 3495 (2010).

53. V. Denisenko, V. Shvedov, A. S. Desyatnikov, D. N. Neshev, W. Krolikowski, A. Volyar, M. Soskin, and Y. S. Kivshar, "Determination of topological charges of polychromatic optical vortices," Opt. Express 17, 23374-23379 (2009). 